\title{
CUIDADO DE ENFERMERÍA EN LA ANGUSTIA ANTE LA MUERTE: NARRATIVA DE ENFERMERÍA
}

\section{NURSING CARE IN ANGUISH OF DEATH: NARRATIVE OF NURSING}

Ramiro Altamira Camacho

Universidad Autónoma de Aguascalientes

Camacho, R. (2020). CUIDADO DE ENFERMERÍA EN LA ANGUSTIA ANTE LA MUERTE: NARRATIVA DE ENFERMERÍA. Revista Ene De Enfermería, 14(3). Consultado de http:// www.ene-enfermeria.org/ojs/index.php/ENE/article/view/1067 


\section{Resumen}

Se muestra una narrativa de situación de enfermería como posibilidad metodológica para el desarrollo científico disciplinar de la enfermería, como ciencia del cuidado. Se toma como referencia principal para su desarrollo lo propuesto en la obra la experiencia vivida por un enfermero en el domicilio del paciente, luego de acudir a una llamada telefónica. Los cuidados ahí realizados trascienden a un contrato establecido y a una dimensión psicofísica. Se observa un cuidado integral. Este cuidado aborda el concepto de la angustia que viven la familia y el paciente, también es posible ver en el mismo enfermero que la narra, un visión integradora y unitaria de su cuidado. En donde su mismo cuidado e intervenciones buscan hacer frente al fenómeno de la muerte que se avecina en el entorno familiar, situando así su propia experiencia y con ello pintando una situación para el aprendizaje de la enfermería con esta visión filosófica. Al final, se hace referencia a dos estudiosas de la disciplina, Jaqueline Fawcett y Barbara Carper, ubicando sus conceptos en la interacción del enfermero con el paciente y la familia, desde una perspectiva humanista. Se concluye que las narrativas, a través del uso de estos referentes teóricos permite el desarrollo reflexivo y crítico de la disciplina de enfer- mería, como ciencia humana, así como el uso y comprensión de los fenómenos de interés

\section{Palabras claves:}

Enfermería; Conocimiento; Narrativas; Cuidado; 


\section{Abstract}

A narrative of the nursing situation is shown as a methodological possibility for the disciplinary scientific development of nursing, as a science of care. The main reference for its development is the experience lived by a nurse at the patient's home, after attending a phone call. The care carried out there transcends an established contract and a psychophysical dimension. Comprehensive care is observed. This care addresses the concept of anguish experienced by the family and the patient, it is also possible to see in the same nurse who narrates it, an integrative and unitary vision of their care. Where their own care and interventions seek to face the phenomenon of death that is looming in the family environment, thus situating their own experience and thus painting a situation for nursing learning with this philosophical vision. In the end, reference is made to two scholars of the discipline, Jaqueline Fawcett and Barbara Carper, locating their concepts in the interaction of the nurse with the patient and the family, from a humanistic perspective. It is concluded that narratives, through the use of these theoretical referents, allow the reflective and critical development of the nursing discipline, as a human science, as well as the use and understanding of the phenomena of interest.
Keywords: Nursing; Knowledge;

Narratives; Care; 

CUIDADO DE ENEERMERÍA EN LA ANGUSTIA ANTE LA MUERTE: NARRATIVA DE ENFERMERÍA

\section{INTRODUCCIÓN}

La narrativa constituye un género en el cual las características se expresan a sí mismas sin otro límite que el impuesto por el mismo autor. El presente trabajo pretende, desde un análisis de una situación de enfermería, profundizar y fortalecer a la enfermería como una disciplina profesional y una ciencia humanista (1-3). Lo que implica sujetar el conocimiento desde una estructura filosófica, es decir, repensar a la enfermería como disciplina (2) y como ciencia desde el vértice de la epistemología (2,4), a decir, desde una filosofía humanista (4), en tanto que es ciencia humanista con un pensamiento humanista (5) y, con ello una postura ética y estética del actuar profesional de la enfermería. A partir de lo propuesto en la guía para el análisis de situaciones de enfermería desarrollada por el Grupo de Cuidado de la Universidad Nacional de Colombia, se tomó la narrativa de una experiencia de la práctica clínica de cuidado domiciliario, para identificar los componentes teórico-conceptuales de la disciplina profesional a la luz de los planteamientos de la enfermería como cuidado (3).

La narrativa aborda el concepto de la angustia, entendido este también como sufrimiento espiritual (6,7). Plantea un fenómeno vivido por Javier, un enfermero peculiar que acude al llamado de una señora, esposa del que en esta situación es el enfermo. Sin embargo, dadas las características personales de Javier su cuidado trasciende la dimensión psicofísica del cuidado interpersonal al Señor Enrique, el paciente.

La angustia es vivida por las personas en el momento próximo a la finitud de la existencia, y se presenta aunada al desamparo y a la desesperación (8). La angustia puede ser entendida también como sufrimiento espiritual $(6,7,9,10)$. En situaciones de cronicidad y muerte la necesidad de abordar la dimensión espiritual se hace patente por parte del ser cuidado, y quien ha sido destinado a cuidar no debe pasar por alto esta emergencia de quien sufre $(11,12)$.

Por lo tanto, se considera importante iniciar este trabajo con una introspección que permita hacer un abordaje filosófico, teórico y práctico. Partiendo de la necesidad de reconocer ampliamente el foco disciplinar, es decir, su objeto, el cuidado y su sujeto de cuidado, así como a la enfermera misma $(4,5)$. Entonces se aborda el conocimiento de sí mismo, del ser, en tanto ontología, del conocimiento científico y su método desde una epistemología (3-5), y las acciones humanas desde el contexto ético, y finalmente una reflexión en cuanto a la estética y su trascendencia en la ciencia, por contradictorio que pareciera. Esto a partir de 
una renuncia formal al paradigma naturalista $(1,3)$ para comprender al ser humano y su cuidado desde aspectos de las ciencias humanas. Esto se verá reflejado en el apartado de los hallazgos, que permite llevar a cabo tal introspección hacia la práctica y la teoría del cuidado de la experiencia de salud.

\section{Posteriormente para conocer y} comprender lo anteriormente mencionado, Carper, de cierto modo, nos ha facilitado ya la reflexión teorizando al respecto, propiamente dicho, con los patrones de conocimiento de enfermería $(13,14)$; nos permite vincular los procesos propios de la enfermería, fenómenos muy abstractos (14), con la teoría y, de igual manera, con la práctica. De una u otra forma se tiene las herramientas (15,16), por así decirlo, para el desglose de y construcción del conocimiento, la narrativa de situaciones de enfermería (17-21).

A continuación, se muestra el ejemplo de una y como es útil tanto para vincular los conceptos metodológicos $(4,15,16)$, teóricos y filosóficos de enfermería $(5,13,14)$, partiendo de las experiencias vividas (3), significados y entendimiento de un mundo tal como se presenta.

Se ubica la narrativa en los patrones de conocimiento de enfermería (18), a las visiones $(2,3,14,19)$ del mundo para enfermería, propuesta por Fawcett (13) y se describen los elementos princi- pales que debe llevar una narrativa, tomando lo expresado en el ejemplo.

\section{DESARROLlo}

"El señor Enrique, un empresario reconocido, de 36 años, trabajaba en la Secretaria de Turismo del Estado, reconocido en el área por su carisma empático, sencillez y alegría peculiar. Hombre de familia, padre de tres niñas una de diecisiete años y gemelitas de cinco, su esposa de la misma edad, profesora de una universidad pública. Originario de la capital se mudó a los 20 años para hacer su vida con su esposa y su hija de apenas dos años, el tiempo les remuneró los esfuerzos de más de diez años y finalmente ambos tenían un algo por lo que levantarse cada día.

Yo, un enfermero con experiencia clínica domiciliaria a personas en procesos de muerte, es decir al final de la vida, debido a que había recibido formación en el manejo de cuidados paliativos.

Cierto día me llaman para que vaya a valorar al señor a su domicilio y especificara al familiar qué cuidado en si podía tener, ya que hacía un año que le habían extirpado un tumor de la cara interna del muslo "no sabían si este era cancerígeno". Al llegar a su domicilio, previa presentación mutua marcamos las condiciones de trabajo, dado que ese era mi fin, me dijo que mi trabajo valía el precio que yo le pusiera pues no a cualquiera estaría en mi lugar y "no cualquiera haría lo que yo". El señor Enrique tenía ya postrado en cama al menos tres meses, físicamente se observaba cansado, agitado, no podía hablar, su garganta estaba reseca al igual que sus labios, su mirada era penetrante se dirigía hacia mí. Tenía en la cara interna del muslo una herida que se mantenía abierta para que cerrara por tercera intención ya que estaba infectada, el diámetro de esa pierna probablemente si era mayor a los cien centímetros de tan edematizada que estaba, un 
edema de tres cruces. Podía valorar a simple vista que el Señor Enrique estaba en condiciones higiénicas poco favorables pues su esposa no podía realizar ciertas actividades por sí sola, como el bañarlo o cambiarle la cama. Refería que, en las últimas noches, tres para ser exactos, había estado con fiebre incontrolable y que la última semana el dolor de la herida era insoportable, no toleraba ni el viento mismo. Era poca la tolerancia a la comida, esta no le manifestaba sensación placentera al gusto y ya de por si era muy poca cantidad. Orinaba en un recipiente que le acercaban de urgencia en cada micción, derramando la orina sobre la herida expuesta.

Al parecer ya había estado internado varias veces en un hospital público, en esta ocasión lo dieron de alta porque "ya no había nada más que hacerle" se le había dado medicamento opiáceo para el manejo del dolor, antinflamatorios no esteroideos, así como la recomendación de que estuviese bien hidratado (refería una sed insaciable).

Podían observarse en toda la habitación imágenes de santos católicos, un par de biblias, $y$ un cristo colgado de su cabecera con un rosario, era más que evidente que el señor es católico. Por lo que le pregunte si ya había recibido la unción de enfermos y/o la comunión, él me dijo que no pero que le gustaría.

Así pasó una semana, yo buscando el confort y la comodidad para el señor, cumpliendo con mi tiempo y horarios acordados. El lunes siguiente al llegar a su domicilio e inspeccionarlo a través de la valoración y auscultación observé que el Sr. Enrique tenía signos de choque, se encontraba inconsciente, de inmediato lo conversé con la esposa para que consideráramos su intervención en un hospital o que el médico tratante le valorara la condición clínica ya que mis cuidados eran limitados por la situación, se rehusó y dijo que en el hospital no le harían nada, que los paramédicos no se lo llevarían que lo mejor sería que se quedara aquí. Dada la situación y, considerado desde mi juicio crítico, intervine para mitigar el choque con medidas no farmacológicas, por lo menos, elevándole las piernas pude mejorar el gasto cardiaco y con esto la recuperación de la tensión arterial y la frecuencia cardiaca, pasados 30 minutos el Sr. estaba nuevamente con un mejor estado de conciencia. Me refería intenso dolor que pese al fármaco no remitía.

Mi labor como enfermero no sólo sería eliminarle el dolor, sino facilitar el afrontamiento y mitigar el sufrimiento espiritual que ahí podía observarse, no sería ya solo bañarlo, afeitarlo y cambiarle la cama, debía hacer uso de otras habilidades para ayudarle, ya que ni los médicos a cargo querían aliviar este dolor físico, mucho menos el espiritual. Sé perfectamente que soy enfermero y conozco muy bien mis competencias pero no por ello puedo dejar a un ser humano sufrir de manera tan desalmada (me decía a mí mismo), sabía que los picos febriles que lo llevaban a la inconciencia, o una de dos, eran por el efecto del tumor (que a estas alturas, según lo expresado por la señora tenia metástasis en peritoneo, hígado y cerebro, pero no le quería decir a su esposo) o era por una cruel infección en la herida, necesitaba administrarle un antinflamatorio que fungiera como antipirético y un antibiótico, ya que eran largos los eventos febriles que no remitían con medios físicos, consulte a una amiga, médico (que se encontraba residiendo en la ciudad y que podía ayudarme por su condición de vocación y servicio, es religiosa), me hizo la receta del antibiótico (dado que actualmente no existe un formato para la prescripción farmacológica por enfermería) y la fiebre se pudo controlar, el antiinflamatorio no tuve problema en la prescripción, así que lo conseguí y lo ministre. 
Esto le dio más tranquilidad a la Familia al ver que remitían los cuadros, jamás les di esperanza en su recuperación, eso no me competía, antes bien me permití facilitar la expresión de sentimientos, emociones, el llanto y que vivieran un duelo, en ese momento me convertía en un pilar emocional para la familia dada la situación que ivían, sabía que yo tenía que ser fuerte pues yo mismo estaba conmovido.

Mis convicciones religiosas siempre se han mantenido al margen de las de los pacientes, no por ello negaba las de ellos así que busque al sacerdote ungió los óleos santos se confesó, y comulgo con mi ayuda ya que la eucaristía tenía que disolverla en agua y dársela con una jeringa (acto permitido para la fe católica). Me convertí ahora en un respaldo no solo emocional sino espiritual, buscando reconfortar al Señor Enrique aliviando el sufrimiento de su espíritu, preparando su alma para despedirse de su familia y a su familia despedirse de él, preparar un paso en la brecha importante en la trascendencia del hombre $y$ eso a mí me daba fuerzas para mantenerme con la postura y la prudencia debida. Así que a partir de ese momento mi centro de atención había ampliado el diámetro, no solo era el señor Enrique, no solo era su fiebre y su dolor, era también su comodidad, su sufrimiento espiritual, su miedo y también estaban sus hijas y su esposa, su madre y su hermano, sus suegros y cuñados quienes sufrían al verlo, sabía que tenía que apoyarlos en esta situación.

Llego el segundo fin de semana, esta semana había sido muy pesada. El sábado tenía que salir de la ciudad y no me atrevía a dejar a la familia sola dos días, todo podría pasar. El señor cada día se veía más deteriorado clínicamente, con un aspecto que en clínica llaman "agónico" pero con una confianza y fortaleza espiritual notoria, al igual con sus hijas y su esposa.
No podía dejar que sus niñas vieran como aquel hombre robusto, fuerte y alegre se retorcía de dolor minutos antes de su muerte, era tan evidente el temor que las niñas sentían ante la muerte, que pensaban que siempre morir seria así de doloroso, tampoco podía con la angustia de su esposa al verlo sufrir, ni siquiera limitarle y evitar que estuviesen en el momento que su padre muriera, solo para que no se "traumaran", pues sabía que podría ser peor

Hable con la familia, les explique del manejo en las personas en este estado y lo crucial e importante que es el que aplique una dosis más de opioide, pues las condiciones en las que se encontraba no estaba para dejarlo morir así, les comente que el medicamento produciría tal alivio al dolor que incluso podría sentir plenitud, pero que también empezaría con dificultad para respirar, es decir, haría un paro respiratorio, pero antes caería en un sueño profundo y esa impresión aparatosa de morir desaparecería. Lo consultamos vía telefónica con una especialista en algología. Finalmente fue lo que convenció a la Señora y la orilló a ir por el medicamento para "aliviar el dolor y limitar, más que la agonía, el sufrimiento".

Básicamente sentía que mi labor en ese momento era dar fortaleza a la familia y no dejar que el Sr. Enrique muriera solo en su propia casa. Cerca de las 19:30 horas su esposa fue por más medicamento, pues cada vez estaba más irritable por el dolor y su aspecto era cada minuto con más énfasis en agonía, me era natural no querer dejarlo sentir más tal sufrimiento, ni a su familia misma, de por sí ya era suficiente con el duelo por la enfermedad y la aceptación de su muerte como para darle horas de dolor, sus últimas horas dentro de ese cuerpo y con dolor. Me quedaba solo la dosis suficiente para remitir por completo ese malestar físico que achacaba al señor Enrique, sabia claramente que podía pasar si yo le aplicaba esa 
dosis, según la prescripción del algologo, para que "dejara de sentir dolor".

Esclarecida la situación y a sabiendas de que la responsabilidad de todo el entorno $y$ ambiente circundante dependería de mis acciones le apliqué esa dosis, la que le tranquilizó y por la que se pudo observar en el señor Enrique la expresión de gratitud y bienestar, permitiendo así un desenlace menos caótico y aparatoso. Me percate que dentro de su tan marcado patrón respiratorio podía auscultarle un tórax congestionado y a través de la orofaringe se podía visualizar una secreción color marrón que, desde mi perspectiva le obstruía la vía aérea y podría encausar el paro respiratorio.

No fue necesario aspirarle (de por si no tenía el material para hacerlo, una jeringa no alcanzaba), el señor estaba en un estado de inconciencia, su pulso muy débil y bradicardico, por cada seis respiraciones que daba presentaba una pausa de cinco segundos y está cada vez se prolongaba, me permití decirle a la hija mayor que le marcara a su mamá y le dijera que ya no sería necesario el medicamento, que regresara a casa para que estuviese en el acontecimiento. Seguido el acto le pedí que se le acercara a su papá, lo abrazara y le dijera cuanto lo amaba y como se sentía en ese momento, cuáles eran sus más grandes emociones y que le santiguara y le diese la bendición como su hija que es. En ese momento la anime a que tranquila que su papá lo estaba, y me aparte quedándome dentro de la habitación (observando la situación y el control que tenía sobre esta). Cuando note un silencio en la chica, me aproxime a ambos y los abrace mientras palpaba así el pulso del señor, hasta que deje de sentirlo, mis palabras hacia la joven fueron "tu Papá ha entrado en la presencia eterna de Cristo, ahora el cuidara de ti y vera que nada te falte jamás”...
Mientras los familiares esperaban en el resto de la casa, llego la señora, con una serenidad que reflejaba conformidad y paz, abrazo a su esposo y a su hija y le agradeció, bendijo y prometió por su amor e hijas, naturalmente se inició en Ilanto, junto a su hija. El resto de la familia parecía estar abrumada por el hecho, sin embargo, la esposa y la hija pudieron proyectar su calma.

Salieron madre e hija tranquilas y satisfechas por el hombre que acababan de despedir, conscientes de que estaba en un lugar donde nunca le faltaría nada, un lugar donde descansaría tranquilo y por la eternidad. Permitimos el paso a los familiares y se les reconfortó en la perdida, permitiendo también expresar y vivir un duelo.

La expresión de cada uno de ellos y su gratitud hacia mi trabajo fue tan gratificante, $y$ recompensable, sabía que lo que esas semanas me habían pagado por prestar un servicio, jamás se pagaría con el estado de tal afrontamiento y sentido de bienestar espiritual que la familia me hizo saber, las lágrimas no se lograron contener, pues sabía plenamente que mi servicio era con ese sentido humano buscando en él la trascendencia misma de lo que yo podía hacer al cuidar del señor Enrique, de su familia y de mí"

\section{Ubicación de la narrativa de} enfermería (3) con las visiones del mundo de enfermería.

Antes que nada, cabe mencionar la aportación de J. Fawcett (13,22) a la enfermería en el sentido de identificar tres formas de ver el mundo, a decir, visones filosofías de enfermería.

Fawcett, desde una posición crítica respecto a los cuatro fenómenos paradigmáticos propios de la disciplina, 
mantiene que de entre las premisas relacionadas con las características que debe conservar todo metaparadigma es necesario diferenciar el conocimiento de este al de otras disciplinas (13,22,23). Por lo que se debe tener bien claro, si se pretende fortalecer a la enfermería como ciencia humanista, que enfermería requiere de otros enfoques y formas de ver el mundo, es decir, "visiones de enfermería" dentro de su modelo de abstracción. Modelo en el cual se desliza el conocimiento de enfermería, partiendo de lo abstracto hacia lo concreto: desde los fenómenos paradigmáticos (o metaparadigma), hasta indicadores empíricos, estos que permiten el desarrollo practico de dicho conocimiento $(2,3,13,20,23-26)$.

La situación narrada por el enfermero permite identificar el arte de cuidar en la simultaneidad del ser humano $(3,18-21,23,26) \mathrm{y}$, mientras el sujeto de cuidado se enfrenta al proceso de finitud de la vida, se percibe como un ser unitario. La perspectiva de enfermería como disciplina de cuidado (27) y como ciencia humana permite observar la manera en la que se integra el conocimiento $(3,18,21)$.

Por un lado, desde la perspectiva de cuidado, se observa la respuesta del enfermero a la necesidad implícita no solo del señor Enrique, sino de toda la familia, e inclusive su propio llamado a servir y dar más allá de la atención clíni- ca y la relación fáctica solamente, así pues, se observa como el enfermero también crece luego de poder ver el resultado obtenido reafirmándose como persona desde de sus propios valores, permitidos a partir de la relación mutua con la familia y el señor en su lecho de muerte. Por otro, como ciencia humana $(4,13,14)$, el enfermero sabía el padecimiento que aquejaba al señor Enrique, tenía conocimiento sobre el proceso fisiopatológico así como las condiciones clínicas y los efectos farmacológicos de los medicamentos necesarios para la enfermedad y sus complicaciones, sin embargo asume como necesaria la comprensión de la experiencia propia de la esposa y las tres hijas, comprende la necesidad de expresar la religiosidad tanto en él como con la familia, surgiendo así una intersubjetividad que permite la trascendencia del conocimiento como una ciencia humana. Permitiendo de esta manera ubicar la narrativa en una visión del mundo de simultaneidad y transformación.

A continuación se muestra como la narrativa de la situación presentada se ubica en una de las visiones del mundo expuestas al inicio de este estudio, unitaria transformativa $\mathrm{o}$ de simultaneidad $(2,3,20,22-24)$

Escuela filosófica - origen: postmodernista, Unitaria-transformativa 
(Newman)(3), de acción simultánea (Fawcett) (3,13,22).

Entidad y cambio: en la situación narrada se puede observar cómo, inmediatamente, la relación del enfermero "y" el paciente se limita solo a un trato laboral y, casi instantáneamente, mientras se observa el ambiente en el que se encuentra el paciente la disposición del enfermero da un giro absoluto. El cambio es simultáneo al ver al paciente como un todo en conjunto pues su fe e idea de trascender en su religiosidad es muy marcada y para ambos es un factor de progreso y cambio importante. De ahí que a partir de este momento pasa de ser enfermero " $y$ " paciente a ser "enfermero-paciente"

Posterior a este cambio cómo se le confiere al paciente más que las necesidades fisiológicas, la necesidad de una estabilidad espiritual, ya que su estado de salud era terminal, lo que reforzaba su acercamiento de su interior con el ambiente y con la creencia en esa fuerza superior, su Dios. Por otro lado, el enfermero quien, también como persona, tenía bien definido quien era, que quería y que estaba dispuesto a dar por sí mismo y para el paciente: debido a la condición espiritual, que fue fortalecida en sus valores y religiosidad, compartida con el paciente.
Esto permitió una relación íntima y mutua entre el paciente, el enfermero y la familia, así como con quien acompañaba al enfermero, permitiendo ese cambio hacia la simultaneidad y muto entre todas las personas ahí vistas como un todo, enfermero-paciente, paciente entendido como el sujeto de cuidado que en este caso es el Sr. Enrique y su familia, haciendo lograr el cambio.

EL enfermero, Persona: dada la situación en la que se describe al paciente se puede ver cómo Javier mantiene las dimensiones en una sola relación con el entorno, lo que originó el cambio. Haciéndose ver como un ser unitario que busca esa armonía en el entorno por completo. Se aprecia en la narrativa la manera en que se expresan los valores y esos sentimientos hacia la familia, su Dios y a su misma muerte (por ser algo específico), manteniendo una relación de él (como enfermero) con el entorno buscando así y en si un giro en su vida, cambiando el curso y el significado de su misma muerte. El enfermero, claramente se deja llevar por lo que le es conferido como persona y a su vez lo reconoce en lo otro a quien también le dignifica, dándole el valor a la historicidad personal del Sr. Enrique. 


\section{Cuidado}

Como un rasgo humano: acciones humanitarias. El enfermero no solo conoce sus implicaciones y funciones técnicas como tal, sino que además, comprende, entiende y se identifica con las necesidades de la familia y del Sr. Enrique.

Como un imperativo moral: dignidad e integridad del individuo. El enfermero atiende al llamado telefónico, acude al domicilio, y se presenta formalmente ante el Sr y su familia, dejando en claro sus funciones y su rol, en este sentido se muestra transparente. El actuar del enfermero se torna con mayor compromiso moral de responsabilidad cuando se percata de que el $\mathrm{Sr}$ requiere más atención, por tanto, mayor tiempo manteniendo la postura inicial de compromiso.

Como Afecto: interés por la protección y el compromiso. En el momento que se decide continuar con el cuidado y la responsabilidad pertinente, buscando no hacer el mal, sino todo lo contrario, cuando se aborda todo el ambiente de la persona y su familia, se hace visible el compromiso.

\section{Como relación interpersonal:} Se ve como en todo momento se expresa la comunicación verbal y no verbal entre el enfermero, Enrique y su familia, siendo esta recíprocamente respetuosa y responsable, hasta volverse un interés simultaneo por el bienestar.

\section{Como intervención terapéutica:}

A pesar de no recibir ya tratamiento médico para la condición del señor Enrique. El enfermero opta, desde su juicio crítico, realizar intervenciones que estrechen los vínculos afectivos y emocionales de la familia y el señor Enrique. Desarrollando una serie de actividades que influyeron en todos los ámbitos que rodean a la persona incluyendo, por ejemplo, el espacio preciso para interactuar con la religiosidad de la familia y la persona. Mostrando el crecimiento y la trascendencia de la familia y, en el mismo acto, del enfermero.

Enfermería-Valoración de Enfermería: Básicamente solo se contaba con un estuche de diagnóstico para valorar signos vitales (estetoscopio, baumanometro y termómetro), sin embargo, los demás datos significativos y que eran subjetivos, necesarios para la interacción simultánea, recolectados en esta situación, por tanto, no son cuantificables se observa por su carácter subjetivo cómo la narrativa se vincula con el cuidado de la experiencia de salud humana.

Ambiente y relaciones: Un vínculo íntimo entre el ambiente mismo la familia la persona y el enfermero. Dejando de lado los prejuicios marcándose con una igualdad humana única e íntima 
en el entorno. No es posible aislar el ambiente del ser, es irreductible el contexto bajo el cual se da la interacción enfermero-paciente.

Metodologías: El enfoque metodológico primordialmente seria cualitativo si se decidiera hacer investigación, pudiendo ser fenomenológico. De ahí, dar ese giro a la hermenéutica y buscar interpretar la interacción y el actuar dentro del contexto de la situación narrada. Comprendiendo así, el cuidado como un todo, desde los patrones de cuidado, por ejemplo.

Fenómenos: Dentro de la narrativa se puede observar como fenómeno, aquel en el que la familia se encuentra unida por sus creencias religiosas y cómo el enfermero logra reforzar esa espiritualidad, que muchas veces se encuentra amedrentada por visiones mecanicistas y que enfermería poco a poco y muy lentamente ha recuperado en el tiempo. Se contempla el fenómeno de la salud-muerte dentro de un campo mayor, la interacción no solo biológica de la enfermedad en el Sr. sino la complejidad en la que se desarrolla el proceso de morir.

\section{Ubicación de la enfermería en} los patrones de Bárbara Carper

En el trabajo, se describe como la narrativa de situaciones de enfermería puede ser empleada para la reflexión filosófica, para la indagación del conocimiento propiamente disciplinar, como método, así como para la mayor comprensión del desarrollo teórico practico. Para ello se ha considerado ubicar la narrativa dentro de los patrones fundamentales de cuidado, a decir, los cuatro patrones de conocimiento en enfermería propuestos por Carper (2,3,18-21,23,26-28).

Los patrones de conocimiento de enfermería son formas y estructuras que visualizan y ejemplifican la manera de pensar y actuar en relación con los fenómenos propios de la enfermería. En este sentido Carper propone que comprender los patrones es esencial para enseñar y aprender la práctica $(3,24)$.

Los patrones propuestos por Carper permiten ampliar la reflexión y la crítica sobre el saber y el conocimiento para la disciplina de enfermería. Así mismo los patrones fundamentales contextualizan la práctica y permiten dilucidar un cuidado holístico, por parte de quien estudia al cuidado como objeto científico de la enfermería $(23,24)$

A partir de aquí se muestra cómo en la narrativa se pueden identificar los patrones propuestos y así ampliar, como lo menciona la misma Carper, el campo de reflexión y crítica hacia el cuidado de enfermería: empírico, personal, ético y estético son los cuatro patrones funda- 
mentales del cuidado y para el conocimiento de enfermería.

Empírico. Saber cómo es el proceso fisiopatológico en el cual se encontraba el señor permite dar respuesta a estas necesidades. Así mismo conocer la importancia de la religiosidad para fortalecer, de cierto modo, la necesidad espiritual no sólo de él como paciente sino de la familia en su totalidad. A partir de la comprensión de la experiencia vivida se permite dar un cuidado más integral que trasciende a los conocimientos biomédicos y al solo actuar mecánico del profesionista que brinda las acciones de cuidado. Implicando el conocimiento como ciencia humana más allá de actos naturalistas, a partir de intervenciones intersubjetivas del cuidado, un quehacer mutuo por parte del enfermero. Lo que exige la incursión de saberes humanista.

Ético. Las actividades realizadas por el enfermero permiten que en cada intervención se observa de forma implícita y explícita, conductas moralmente comprometidas y comprometedoras. Sin embargo, en la relación enfermero-paciente en que se establece la modalidad y la responsabilidad del "pago", se ve como el enfermero va más allá del aspecto económico por el vínculo libre y responsable que se llega a generar en la interacción. También se observan conductas moralmente comprometidas cuando el enfermero "debe" dar el tratamiento para el control de la fiebre, permitiendo que los valores como la libertad y la responsabilidad sobresalgan. No obstante, aun cuan hubo un dialógo con la familia, no se documenta la voluntad el paciente respecto al actuar en el momento del desenlace, más que en un inicio, cuando el señor se encontraba en un estado de conciencia idóneo. En este punto se puede observar a la familia tranquila y al enfermero decidido a intervenir, tal vez por las palabras que el señor comentó el primer día. Lo que hace el acto de cuidar trascienda al compromiso de cuidar una herida o un "paciente terminal".

Personal. Se observa como las características y los valores que atañen al enfermero se proyectan a partir de la interacción con la familia en general, además el interés en el bienestar de ellos. También permite ver como se concibe a sí mismo como un ser humano que tiene emociones y necesidades ante las diferentes situaciones en las que se ve inmerso durante la experiencia de cuidar al señor Enrique y a su familia.

Estético. El enfermero ve en el señor Enrique, la familia ("lo otro") ese lienzo sobre el cual plasma sus conocimientos, sentimientos y emociones, valores y creencias. Proyecta su ser en otro ser. De igual forma se percibe el detalla- 
do momento en el que cada acción que realiza permite transformar el espíritu propio del enfermero en un instrumento para transformar y crear emociones distintas como el miedo en amor, la angustia en esperanza, y la tristeza en paz. El aspecto más abstracto y profundo, posiblemente esencial para el cuidado de enfermería.

\section{CONCLUSIÓN}

Cuando llega el momento de releer y compartir esta experiencia, plasmada en la situación narrada, permite encontrarse con un enfermero que en el pasado se fue (y que, personalmente me implica como profesionista del área de la salud). Permite renacer esas emociones, esa visión que en su momento yo como enfermero sentía y que con el tiempo pude haber enmascarado antes de perder u olvidar. Hay situaciones en las que el ejercicio profesional se ve limitado por institucionalismos o incluso por perjuicios personales y de la sociedad, en este caso la experiencia de cuidado dependía plenamente del enfermero y de manera simultánea de la familia, el único límite era la conducta moral.

La situación anterior muestra como el enfermero apoya al paciente y a la familia a crear herramientas para afrontar la muerte, permitiendo, a partir de su experiencia personal y conoci- miento, la expresión de sentimientos y emociones inherentes al proceso de morir y esencialmente a lo que sigue después de ello, el vacío y la angustia que comúnmente se presenta ante la incertidumbre y el miedo (22).

Esta situación narrada, pues, permite crecer no solo como profesionista sino, de cierta manera más compleja cómo persona, como ser humano y de ahí éticamente como un profesional $(4,21)$.

Desarrollar narrativas de enfermería implica mantener un rigor metodológico $(14,29)$, en tanto que requiere una postura moral y científica de la disciplina. Por un lado, no es fácil descubrir las habilidades literarias en cada uno de nosotros para poder expresar y hacer énfasis en lo sucedido, primero, porque esto no está explícito en el currículo académica de enfermería, segundo porque no todos las y los enfermeras(os) han sido sensibilizados en el uso de narrativas como un método $(4,13,14)$ para el desarrollo disciplinar y científico. Se enfrenta uno tanto al conflicto de lo correcto en la narrativa $(14,15)$ cuando lo correcto y lo justo es en medida de lo posible narrar lo esencial.

Por otro lado, el fortalecimiento de enfermería como ciencia y, más específicamente, como ciencia humana, es posible, como todas las ciencias, a partir de la reflexión y el uso de métodos subjetivos que permitan develar la compleji- 
dad de los fenómenos de interacción humano, propiamente dicho la intervención de cuidar la experiencia de salud humana $(2,13)$ con sus implicaciones en el conocimiento científico y un conocimiento de "lo otro" como lo es lo humano. Ubicar la narrativa en las visiones de Fawcett y lo patrones de Carper, una vez más dan patente de la autenticidad y rigor cualitativo de dicho fortalecimiento disciplinar.

Las narrativas de situaciones de enfermería permiten el acercamiento de la práctica, a la teoría e incluso una reflexión filosófica de la disciplina profesional que es la Enfermería, ciencia del cuidado, ampliando así el criticismo del pensamiento, fortaleciendo la construcción y creación de saberes nuevos para la disciplina.

\section{AGRADECIMIENTOS}

Al señor Enrique, a su familia por permitirme tener esta experiencia como enfermero y como ser humano. A la enfermera que apoyo a desarrollar los cuidados y la génesis de esta narrativa. 


\section{BiBLIOGRAFÍA}

1. Cabal VE, Guarnizo MT. Enfermería como disciplina. Rev Colomb Enfermería [Internet]. 2011;6:73-81. Disponible en : https://dialnet.unirioja.es/descarga/articulo/4036648.pdf

2. Durán de Villalobos, MM. Marco epistemológico de la enfermería [Internet]. Aquichan. 2002. p. 1. Disponible en : http://dialnet.unirioja.es/ $\mathrm{s}$ e $\mathrm{r} v \mathrm{l}$ e $\mathrm{t} / \mathrm{a} \mathrm{r} \mathrm{t}$ i c u l o ? codigo $=2107428 \&$ info=resumen\&idioma $=$ SPA

3. Gómez Ramírez O. La situación de enfermería: fuente y contexto del conocimiento de enfermería: la narrativa como medio para comunicarla. Umiversidad Nacional de Colombia, editor. Colombia; 2010. 260 p.

4. Morin E. Introduccion al pensamiento complejo. Gedisa; 2009. 176 p.

5. Ortiz LB, Carreño SP, Carrillo GM, Díaz LC, Herrera BS. Analisis epistemológico y ontológico de la práctica de enfermería en personas con cancer y sus familias. Salud Uninorte. 2014;30(2): 210-6.

6. Simão TP, Chaves ECL, lunes DH. Spiritual distress: the search for new evidence. Rev Pesqui Cuid é Fundam Online [Internet]. 2015;7(2):2591. Disponible en : http://seer.unirio.br/index.php/cuidadofundamental/article/view/3369

7. Cássia E De, Chaves L, De EC. Spiritual distress : integrative review of literature Angústia espiritual : revisão integrativa da literatura. Online Brazilian J Nurs [Internet]. 2008;7(2). Disponible en : http://objnursing.uff.br/index.php/nursing/article/ view/j.1676-4285.2008.1551/358

8. Sartre JP. El existencialismo es un humanismo. 1st ed. Edhasa; 2009. 128 p.

9. Caldeira $S$, Carvalho EC de, Vieira $M$. Between spiritual wellbeing and spiritual distress: possible related factors in elderly patients with cancer. Rev Lat Am Enfermagem [Internet]. 2014;22(1):28-34. Disponible en : http://www.sciel o . b r / s c i e l o . p h p ? script=sci_arttext\&pid=S0104-1169201400010002 $8 \& \operatorname{lng}=$ en\&tlng=en

10. Muñoz Devesa A, Morales Moreno I, Bermejo Higuera JCa, Galan GOnzalez Serna JM. La Enfermería y los cuidados del sufrimiento espiritual. Index Enferm 2014; 23(3):153-6.

11. Sánchez Herrera B. Dimensión espiritual del cuidado en situaciones de cronicidad y muerte. Aquichan [Internet]. 2004;4(4):6-9. Disponible en: http://www.scielo.org.co/pdf/aqui/v4n1/v4n1a02.pdf

12. Jiménez MO, Vargas MGO, Domínguez AO, Castañeda RFG. Espiritualidad y cuidado de enfermería / Spirituality and nursing care. RICSH Rev Iberoam las Ciencias Soc y Humanísticas [Internet]. 2016;5(10):231-47. Disponible en:
https://www.ricsh.org.mx/index.php/RICSH/article/ view/86/450

13. Fawcett J. Contemporary Nursing Knowledge: Analyisis and Evaluation of Nyursing Models and Theories. 3rd ed. Davis FA, editor. 2012. 480 p.

14. Prado ML, Souza ML, Monticelli M, Cometto MC GP. Investigación cualitativa en enfermería. Metodología y didáctica. 1st ed. OPS P, editor. washinrton; 2013. 276 p.

15. Guerreiro D, Vieira da Silva MT. N arra as como técnica de pesq fatos nada têm a ver com a experiência da da intenção da entrevista . Porém , quando o que as pessoas têm e querem contar mostra- informações a fim de planejar o cuidado e / ou prática pela pesquisa. As análises que , muita. 2002;10(3):423-32.

16. Ruiz Bugarin CL. Narrativa de enfermería: dejando ir a Doña Paula. Ene [Internet]. Grupo de Enfermería ENE; 2015 [cited 2019 Mar 5];9(2):0-0. Disponible en : http://scielo.isciii.es/scielo.php? script $=\mathrm{sci}$ _arttext \& pid = S 1988 - 348 $X 2015000200009 \& \operatorname{lng}=e n \& n r m=i s o \& t \mid n g=e n$

17. Gómez Rojas ML; Rodríguez Díaz BL. Situación de enfermería como herramienta para enseñar el proceso de atencion de enfermería. Rev Cuid. 2013;4(1):544-9.

18. Carper B. Fundamental patterns of knowing in nursing. Adv Nurs Sci. 1978;1(1):13-23.

19. Valderrama Sanabria ML, Peña Pita AP, CLavijo ALvarez LM. Narrativa: El Estudiante De Enfermería Aprendiendo El Arte De Cuidar. Rev Cuid [Internet]. 2017;8(1):1488-98. Disponible en: http://www.scielo.org.co/pdf/cuid/v8n1/2216-0973cuid-8-01-01488.pdf

20. Briñez KA. Narrativa de enfermería : visión y patrones de conocimiento en una entrevista en el hogar. Rev Colomb Enfermería. 2014;9(9):142-8.

21. Palencia IG. Técnica de la narrativa en enfermería, patrones de conocimiento y abordaje teorico. Rev Ciencias Biomed [Internet]. 2012;5(1): 174-9. Disponible en : http://www.revistacienciasbiomedicas.com/index.php/revciencbiomed/article/ view/189

22. Fawcett J. Middle range nursing theories are necessary for the advancement of the discipline. Aquichan [Internet]. 2005;5(5):32-43. Disponible en : http://aquichan.unisabana.edu.co/index.php/aquichan/article/view/57

23. Duran de Villalobos MM. La ciencia, la ética y el arte de enfermería a partir del conocimiento personal. Aquichan [Internet]. 2005; Disponible en : http://aquichan.unisabana.edu.co/index.php/aquichan/article/view/62/129 
24. Chinn P. Kramer M. Knowledge Development in Nursing: Theory and Process. 9th ed. Mosby, editor. 2014. 288 p.

25. J. Grau, M.C. Llantó, C. Masip, M. Chacón, M.C. Reyes, O. Infante, T. Romero, I. Barroso OM. Ansiedad y actitudes ante la muerte: revisión y caracterización en un grupo heterogéneo de profesionales que se capacita en cuidados paliativos. Pensam psicológico [Internet]. 2008;(10):27-58. Disponible en : http://Odialnet.unirioja.es.cataI e g.uoc.edu/descarga/articulo/ 2792657.pdf\%5Cnhttp://0-dialnet.unirioja.es.cataleg.uoc.edu/servlet/extart?codigo=2792657

26. Landeros Olver EA.. Identificación de los patrones de conocimiento en un incidente crítico. Horiz enfermería. 2002;(13)1-7.

27. Afanador NP. EL cuidado como objeto del conocimiento de Enfermería. Av En Enferm [Internet]. :43-51. Disponible en : http://www.enfermeria.unal.edu.co/revista/articulos/xx1_5.pdf

28. Navarrete Borrero AA. Evidenciando el patron de conocimiento estetico a partir de la narrativa de Enfermeria. Horizontes Enferm. 2013;24(1):9-16.

29. Aimar R, Videla N, Torre M. Tendencias y perspectivas de la ciencia enfermería. Enfer Global [internet]. 2006 Nov Disponible: http://revistas.um.es/eglobal/article/view/385 